\title{
Blasenkrebs: Point-of-Care-Tests direkt in der Praxis
}

— Die Früherkennung des Harnblasenkarzinoms ist von großer Bedeutung, denn bei früher Diagnose sind die Heilungschancen heutzutage gut bis sehr gut. Folgende Point-of-Care-Tests stehen dafür zur Verfügung: der NMP22-Test und der BTA-StatTest. Beide Verfahren können direkt in der Arztpraxis durchgeführt werden.

Der NMP22-Test, der im Gegensatz zum BTAStat-Test von der Food and Drug Administration (FDA) der USA für die Primärdiagnostik, das Screening von Risikopersonen und für das Monitoring von Blasenkrebs zugelassen ist, weist das tumorspezifische nukleäre Matrixprotein 22 nach. Der NMP22-Test ist auch bei Hämaturie zuverlässig. Dieses Kardinalsymptom für das Harnblasenkarzi- nom führt beim BTA-Stat-Test oft zu falschpositiven Ergebnissen. Das konnte eine experimentelle Studie zeigen, die Tübinger Urologen im September 2009 auf dem 61. Kongress der Deutschen Gesellschaft für Urologie in Dresden präsentierten.,,Sowohl der NMP22- wie auch der BTA-Stat-Test zeigen eine Spezifität von $80 \%$ bis $96 \%$ unter Berücksichtigung der Ausschlusskriterien. Es ist daher unverständlich, dass die Blasenkrebsfrüherkennung noch so vernachlässigt wird. Die Vorsorge des Harnblasenkarzinoms bei Risikopatienten lässt sich ohne großen Aufwand als IGeL-Leistung in der Praxis umsetzen“, meint Dr. Gerson Lüdecke, Urologe an der Universitätsklinik Gießen. Lüdecke tritt seit Jahren dafür ein, asymptomatische Risikopersonen frühzeitig auf Blasenkrebs zu untersuchen. Die Risikofaktoren des Harnblasenkarzinoms wie beispielsweise langjähriges Rauchen sind gut bekannt. Lüdecke konnte daher den „RisikoCheck Blasenkrebs“ im Internet entwickeln:www.riskcheck-bladder-cancer. info. Ärzten, die sich zur Nutzung anmelden, können auf Basis eines Fragebogens das persönliche Risiko der Patienten ermitteln. Zudem können die Daten zur Verlaufskontrolle und epidemiologischen Grundlagenforschung gespeichert werden.

Nach Informationen von

Matritech, Freiburg

\section{Granatapfel-Polyphenole mit chemopräventiver Wirkung}

- Prostatakrebspatienten haben häufig den starken Willen, ihren Gesundheitszustand aktiv durch Veränderungen ihrer Lebens- und Ernährungsweise zu verbessern. Allerdings ist es für Patienten und Ärzte gleichermaßen schwierig, einen Weg durch den Dschungel der vielfältigen Ernährungsratschläge zu finden. Das Fachbuch „Chemopräventive und ernährungstherapeutische Wirkung von Polyphenolen bei Prostatakarzinom mit Schwerpunkt: Granatapfel-Polyphenole“ von Dr. med. Ludwig Manfred Jacob (ISBN: 9783000304040 ) soll hierbei als eine wissenschaftlich fundierte Hilfe dienen. Der erste Teil des Buches behandelt die komplexe Kanzerogenese des Prostatakarzinoms. Granatapfel-Polyphenole und deren Effekte in der Chemoprävention sowie in der adjuvanten Ernährungstherapie des Prostatakarzinoms bilden den Schwerpunkt des zweiten Teiles.

Nach Informationen von Dr. Jacob's Institut, Tauberbischofsheim

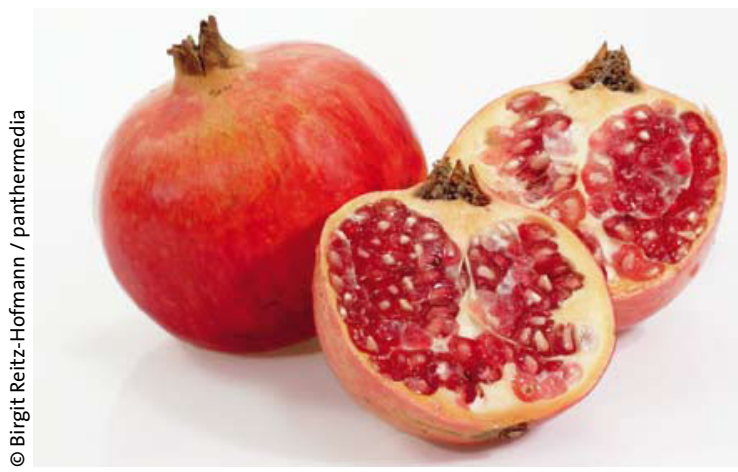

Granatäpfel sind reich an Polyphenolen.

\section{Duloxetin auch bei älteren Patientinnen gut verträglich}

- Ein Mittel der Wahl bei Belastungsinkontinenz ist der Serotonin-Noradrenalin-Wiederaufnahmehemmer Duloxetin (Yentreve ${ }^{\circledR}$ ). Die Beobachtungsstudie „DuRoSa“ (Duloxetin, Routine, Safety) mit mehr als 12.000 Patientinnen belegt nun das allgemein gute Vertäglichkeitsprofil des Wirkstoffs und zeigt zudem: Selbst bei älteren, multimorbiden Patientinnen ist der Wirkstoff in der Regel gut vertäglich - auch bei Komedikation. Eine Arbeitsgruppe der DuRoSa-Studie fokussierte auf Patientinnen, die sich in der Behandlung von Haus- oder Allgemeinmedizinern befanden $(n=5.879)$. Mit durchschnittlich 63,1 Jahren waren die Teil- nehmerinnen deutlich älter als das Patientenklientel der placebokontrollierten PhaseIII-Studien, die zur Zulassung von Duloxetin geführt hatten (im Durchschnitt 52,1 Jahre). Viele Patientinnen litten zudem an Begleiterkrankungen wie Bluthochdruck, Diabetes, Depressionen oder Herzerkrankungen und nahmen eine Komedikation ein. Während der zwölfwöchigen Behandlungsphase erhielten die Patientinnen Duloxetin oder eine nicht invasive Vergleichstherapie. Die Evaluation ergab: Im Praxisalltag ist Duloxetin vergleichbar gut verträglich wie in den kontrollierten klinischen Studien: Nur bei $2 \%$ der Patientinnen aus der Duloxetin-Gruppe kam es zu Nebenwirkungen, die zum Abbruch der Therapie führten. Insgesamt traten in der Duloxetin-Gruppe (9,1\%) etwas mehr Nebenwirkungen auf als in der Kontrollgruppe $(5,7 \%)$. Schwere Nebenwirkungen traten in beiden Gruppen selten auf.

Bei Harninkontinenz kann ein strukturiertes Patientinnenmanagement entlastend für das Praxisteam sein. Wie so etwas aussehen kann, zeigt eine Online-Fortbildung von Lilly Deutschland (Passwort für Ärzte: Zeitgewinn, für medizinische Fachnangestellte: Wissen) auf www.lillyfortbildung.de.

Nach Informationen von Lilly, Bad Homburg 